\title{
Reseña del Libro: Diálogos Psicoanalíticos: Amor, desamor y vínculos de amistad en la contemporaneidad
}

\section{Book Review: Psychoanalytic dialogues: Love, Heartbreak and Friendship Bonds in Contemporaneity}

Antonia Iglesias-Hermenegildo ${ }^{a}$, José Jesús Serrano-Ruíz ${ }^{b}$

\begin{abstract}
:
This book addresses a universal theme: love as well as its counterpart, heartbreak and a bond derived from this affection that is friendship. Within the multiple possibilities to analyze this affect, this work addresses it from a psychoanalytic theoretical perspective. There are 4 axes in which it is divided. The first is about love, heartbreak and social networks, the second section analyzes transference love from the clinical field: the clinic in the new / old social ties; The third section takes up the theme of violence and perversion in the love-heartbreak relationship, the fourth axis includes excess love: on maternal damage and the family relationship, a fifth section is on the paternal bond in contemporaneity, and finally it talks about the bond of friendship: permanence and changes in the XXI century.
\end{abstract}

\section{Keywords:}

Love, heartbreak, friendship, psychoanalysis

\section{Resumen:}

Este libro aborda un tema universal: el amor así como su contraparte el desamor y un vínculo derivado de este afecto que es la amistad. Dentro de las múltiples posibilidades para analizar este afecto, este trabajo, lo aborda desde una perspectiva teórica psicoanalítica. Son 6 los ejes en los que se encuentra dividido. El primero es sobre amor, desamor y redes sociales, el segundo apartado analiza desde el campo clínico el amor de transferencia: la clínica en los nuevos/viejos vínculos sociales; la tercera sección retoma el tema de la violencia y perversión en la relación amor-desamor, en el cuarto eje se incluye el amor en exceso: sobre el estrago materno y la relación familiar, un quinto apartado es sobre el vínculo paterno en la contemporaneidad, y finalmente se habla sobre el lazo de amistad: permanencias y cambios en el siglo XXI.

\section{Palabras Clave:}

Amor, desamor, amistad, psicoanálisis

\section{Introducción}

Este texto forma parte de una colección de libros realizados por el colectivo Canal Freudiano, que reúne un grupo de psicoanalistas de diferentes puntos del país quienes se han cuestionado sobre el tema de la violencia ante el evidente aumento de sus manifestaciones. Considerando lo complicado de este fenómeno, se sabe que una sola comprensión teórica sería insuficiente para abordar el tema es por ello que se hace necesario que diferentes perspectivas teóricas y de intervención se sumen a realizar aportaciones y a plantearse análisis basados en la complejidad. El psicoanálisis ha sido criticado por su singularidad en la intervención pero también se le reconoce la profundidad para analizar diversos fenómenos en los que se pone en juego el psiquismo y la violencia sin duda es uno de ellos, es así que el campo psicoanalítico podría aportar algo para tratar

\footnotetext{
Autor de Correspondencia, Universidad Autónoma del Estado de Hidalgo, https://orcid.org/0000-0002-0525-6852, Email: jesus_serrano@uaeh.edu.mx

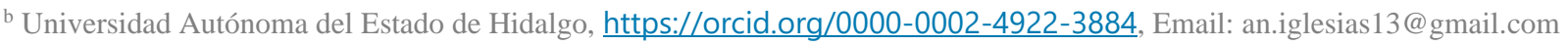


de profundizar e ir repensando el fenómeno que a todos debe preocuparnos y al que no debemos ser indiferentes. Este colectivo propuso a manera real y simbólica "sacar al psicoanálisis del consultorio" y metafóricamente poner en el diván las nuevas complejidades manifestadas en los distintos vínculos, además se propuso un dialogo no sólo intra-psicoanálisis, sino interdisciplinario apuntando a la escucha de otras disciplinas, a fin de contribuir a dilucidar la opacidad de tan complejos fenómenos que se presentan tanto en la clínica como en la vida social.

El trabajo de este colectivo tiene por objetivo dialogar no sólo entre psicoanalistas sino incluir a los universitarios para pensar diversos temas de interés, es así que utilizando el formato de coloquio se ha dialogado sobre la violencia, lo social y lo sexual en el siglo XXI y sobre el amor y desamor.

\section{Desarrollo}

Esta obra realizada por los integrantes de este colectivo reúne los trabajos presentados en el 3er coloquio de Canal Freudiano el cual tuvo lugar en Pachuca, Hgo. en el año 2019 e incluye los 6 ejes que se abordaron durante el coloquio y en cada uno de ellos son incluidos los trabajos que se presentaron y desarrollaron durante el evento.

En el primer eje se toca el tema del amor y desamor y su relación con las redes sociales, se aborda cómo ha cambiado este vínculo a partir del advenimiento de las redes sociales como una forma de "contacto inmediato", pero a su vez han sido un dispositivo que ha alejado la construcción de vínculos profundos, este tipo de relación ha planteado nuevas formas de goce aunque la vida amorosa de los sujetos parece tener los mismos conflictos aunque con un dispositivo diferente, en algunos casos este dispositivo favorece el vínculo en otros lo aparta o complejiza. Se cuestiona si el acontecimiento amoroso no se ha convertido en un espectáculo y deja de tener la intimidad que lo caracteriza.

El segundo tema fue el amor de transferencia en los nuevos vínculos sociales y amorosos, ¿se ha transformado en algo el trabajo en la clínica, o cómo podemos pensarlos en esta contemporaneidad?, la transferencia como instrumento técnico puede ser agente de la cura en el trabajo psicoanalítico o por lo contrario puede resultar un obstáculo cuando no es analizado e interpretado. Se analiza sobre la importancia dentro del trabajo clínico respecto a la supervisión a fin de que en la praxis no se encuentren situaciones de abuso o confusión por parte de ambos actores del trabajo analítico.

En el tercer eje se aborda un tema por demás emergente, la violencia y perversión, en este caso en la relación amordesamor, sin duda, la violencia se puede encontrar en cualquier relación, pero en particular en el lazo del amor cobra un sentido particular pues éste atraviesa por un periodo de idealización que se ve interrumpido por un acto violento. De las distintas formas de violencia, se habla sobre los lazos cada vez más superfluos con temor a la intimidad y al compromiso que dan a paso a distintas formas de violencia, se abordan las diferentes expresiones de la perversidad, el acto perverso y la perversión en sí mismo en el vínculo amor-desamor.

El cuarto eje que se integra en este texto refiere sobre la falta de amor que sin duda es dolorosa y dañina pero su exceso también lo es, es el planteamiento que se realiza en los trabajos de la mesa que se tituló "amor en exceso, sobre el estrago materno y la relación familiar". Así mismo se integran reflexiones en torno a la declinación de la función paterna que da espacio a que el estrago materno se haga presente. En esa relación tan particular el hijo desaparece en relación a la madre por lo que también se aborda cómo será posible la construcción de un camino que permita al hijo un camino diferente en el que se apuntale su deseo.

El quinto eje denominado sobre el vínculo paterno en la contemporaneidad, se incluyen trabajos que dan cuenta del lazo paterno, no sólo por la relevancia para la estructuración psíquica del sujeto sino desde el vínculo particular de ese amor entre el padre y el hijo. Los textos de este apartado incluyen nuevas formas de ejercicio de la paternidad, se expone el papel del padre en el caso de hijos desaparecidos, reflexionando si sólo el lugar de la madre puede albergar un amor que no conoce límites. Se cuestiona si se le ha asignado al padre un nuevo lugar distinto al del padre tradicional de los 50's y como la función paterna es estructurante en la vida del sujeto.

En el último apartado El lazo de amistad: permanencias y cambios se cuestiona si este tipo de vínculo ha cambiado o hay un ocaso de ambos a partir de que se han diluido las líneas entre el amor y los vínculos de amistad. Se proponen un análisis respecto a si las redes sociales han modificado y qué medida este tipo de relaciones, sin que ello implique sanatizar estas vías de comunicación sino de señalar su influencia en la contemporaneidad de los lazos sociales.

A manera de cierre consideramos que este texto aborda temas por demás actuales e interesantes, el amor y por ende el desamor son sentimientos que están presentes desde siempre, lo importante es pensar de qué manera y cómo se juega en la constitución de los vínculos contemporáneos.

\section{Referencias}

[1] Iglesias A, Serrano J. Diálogos Psicoanalíticos. Amor, desamor vínculos de amistad en la contemporaneidad. México: El diván negro; 2021. 